\title{
Evidence for transmissibility of Alzheimer disease pathology: Cause for concern?
}

\author{
Michael B. Coulthart PhD, Gerard H. Jansen MD, Neil R. Cashman MD
}

Competing interests: None declared.

This article has been peer reviewed.

Correspondence to: Michael Coulthart, michael coulthart@phac-aspc.gc.ca

CMAJ 2016. DOI:10.1503 /cmaj.151257
$\mathrm{R}$ ecently, a research group from University College London examined the brains of eight patients, all of whom had died after contracting Creutzfeldt-Jakob disease (CJD) through treatment with prion-contaminated growth hormone prepared from donated human pituitary tissue. ${ }^{1}$ The researchers found marked amyloid- $\beta$ plaque pathology characteristic of Alzheimer disease in these patients, which re-energized debate on an old question: Could Alzheimer disease arise following "prion-like" transmission of protein pathology between individuals?

Neurodegenerative diseases, many of which lead to dementia, present a major health challenge worldwide. Given, for example, the projected fourfold increase in the global prevalence of dementia by 2050 through population aging, emphasis is being placed on mitigation of medical, social and public health impacts. ${ }^{2}$ This need, combined with the complexity of the biological processes underlying dementias, has inspired a dynamic program of biomedical research into neurodegenerative diseases for several decades. Arguably, the single most incisive resulting insight is the recognition that pathologic behaviour of specific proteins can have diverse toxic effects, to which neurons seem particularly vulnerable. ${ }^{3}$ The term "proteinopathies" denotes diseases involving such proteintriggered pathology, including Alzheimer disease,

\section{KEY POINTS}

- Many neurodegenerative diseases, such as Alzheimer disease and Parkinson disease, are proteinopathies that are caused by pathologic misfolding and aggregation of disease-specific proteins.

- Prion diseases, including Creutzfeldt-Jakob disease (CJD), were long considered the only proteinopathies transmissible between individuals by an infection-like mechanism; however, other proteinopathies have recently been reported to be induced by experimental inoculation of animals with diseased tissue.

- Debate about possible implications for human health was intensified by a recent report of several young patients in the United Kingdom, who contracted CJD from contaminated cadaveric human growth hormone and who unexpectedly also had brain pathology characteristic of Alzheimer disease.

- The likelihood that Alzheimer disease could be spread directly between humans through a prion-like transmission process is presumably low; however, continued research and risk assessment, particularly on decontamination of medical instruments, would be appropriate.
Parkinson disease, Lewy body dementia and amyotrophic lateral sclerosis. ${ }^{3}$

Two key features of this molecular misbehaviour - protein misfolding and aggregation - are linked to the disruption of physiologic functions that maintain a cell's proteins in a state of homeostasis known as proteostasis. ${ }^{3}$ Persistent misfolding and aggregation typically lead to formation of microscopically visible protein deposits in brain tissue. These deposits, which can be intracellular or extracellular, appear as amorphous plaques or as crystal-like "amyloid plaques" formed by the aggregation of threadlike structures (fibrils) consisting of ordered arrays of misfolded protein. This phenomenon has aided in the classification and diagnosis of proteinopathies and continues to guide research on their causes.

Creutzfeldt-Jakob disease has long been considered unique among the neurodegenerative proteinopathies because of its infection-like transmissibility between individuals. This capacity is attributed to the ability of the misfolded prion protein $\left(\mathrm{PrP}^{\mathrm{Sc}}\right)$ to act as a template for further misfolding of normal $\operatorname{PrP}\left(\operatorname{PrP}^{\mathrm{C}}\right)$ (Figure 1A). The term "prion" was coined to describe the implied proteinaceous infectious particle. ${ }^{4}$ Epidemic spread of CJD (e.g., through invasive medical procedures) has been well documented. ${ }^{5}$

Intriguingly, recent studies have extended the prion concept to other misfolded prion-like proteins that propagate their misfolded state within an individual. ${ }^{6}$ Indeed, the tissue pathology characteristic of several such proteinopathies can be induced in genetically modified or normal experimental animals by inoculation with diseased human or animal brain tissue. ${ }^{7}$ Among these pathologies are plaques composed of a peptide (amyloid- $\beta$ ) that are found in large numbers in Alzheimer disease and are believed to accumulate asymptomatically decades before clinical onset. Amyloid- $\beta$ plaques are accompanied in Alzheimer disease by aggregates of another misfolded protein, the microtubule-associated protein tau, which has also been transmitted to animals by inoculation. These findings merit consideration of the potential implications for human health; however, interpretation has remained difficult. ${ }^{7}$ 


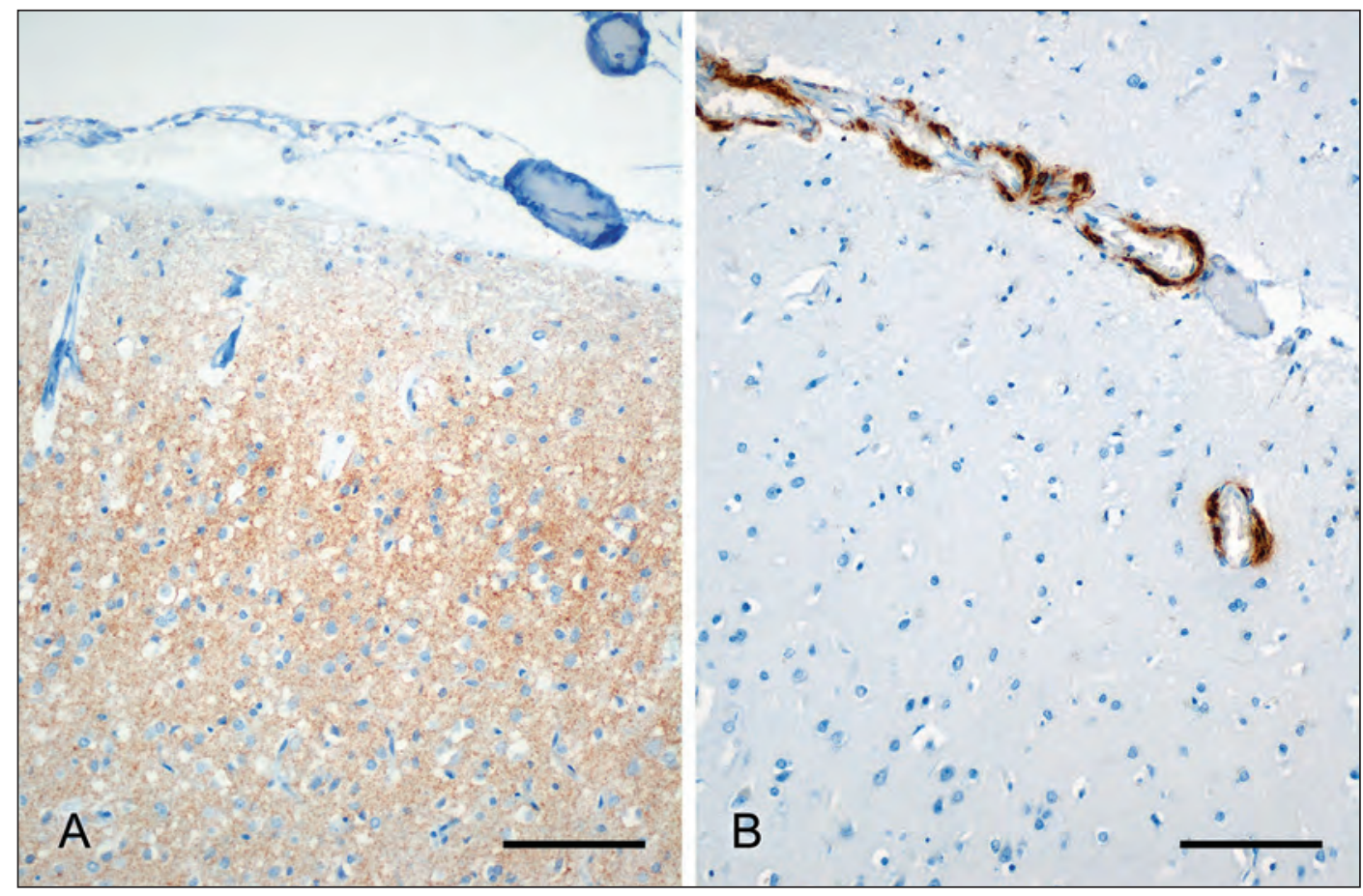

Figure 1: Photomicrographs of neocortex tissue in degenerative proteinopathies (black bars represent $100 \mu \mathrm{m})$. Immunohistochemical staining (brown) was used to show (A) misfolded prion protein, with fine, granular ("synaptic") deposits throughout the neuropil; and (B) amyloid- $\beta$, with amyloid angiopathy in blood vessel walls in the arachnoid and superficial neocortex.

A recent study from researchers at University College London has brought renewed focus to this discussion. ${ }^{1}$ This group examined the brains of eight patients, aged $36-51$ years, who had died of CJD after receiving treatment with prioncontaminated growth hormone sourced from donated human pituitary tissue. In addition to deposits of $\operatorname{PrP}^{\mathrm{Ps}}$, the brains of seven of these patients exhibited marked amyloid- $\beta$ plaque pathology. In four patients, the plaques were concentrated around blood vessels, qualifying the pathology as cerebral amyloid angiopathy (Figure 1B). Interestingly, tau deposits were not found. The patients were screened for known genetic risk factors for Alzheimer disease, with uniformly negative results. These findings prompted the authors to suggest that the amyloid- $\beta$ pathology may have been induced in these patients by a prion-like mechanism, through co-contamination of the growth hormone preparations from which they contracted CJD. As noted by the authors, the findings may represent the first evidence for such transmission in humans, despite negative epidemiologic findings in several previous studies (see citations in the article by Jaunmuktane and colleagues ${ }^{1}$ ).

These observations are unlikely to have arisen by chance. Although co-occurring Alzheimer disease pathology is not uncommon in CJD, it is much more frequent in older patients. ${ }^{8}$ Quantitatively, the odds against such a coincidence are about 12 000:1, assuming 3\% prevalence of amyloid- $\beta$ angiopathy among people 36-51 years of age without Alzheimer disease. ${ }^{9}$ This leaves two other possibilities - coinfection, as suggested by the authors, or a secondary effect of the CJD process that promoted deposition of amyloid- $\beta$. Evidence is not lacking for candidate mechanisms of the latter sort - in addition to disruption of proteostasis, it is possible, for example, that $\mathrm{PrP}^{\mathrm{Sc}}$ impairs extracellular clearance of amyloid- $\beta$ or that direct molecular cross-templating occurs. ${ }^{10}$ Any such alternative hypothesis, however, must be consistent with the unexpectedly intense amyloid- $\beta$ pathology found in four of the patients with iatrogenic CJD, as well as the age-specific distribution of amyloid- $\beta$ pathology in all patients with CJD. Further research is needed to address these questions.

What would be the implications of a confirmation that pathology resembling that of Alzheimer disease can be transmitted between humans, in at least some circumstances? With discontinuation of cadaveric sourcing of human growth hormone in Canada in April 1985 and the availability of synthetic products in January 1986, this particular iatrogenic threat has been eliminated. Although four cases of iatrogenic CJD associated with dura mater transplantation have been identified in Canada to date, fortunately no cases associated with growth hormone treatment have been found. ${ }^{11}$ As 
emphasized by the authors of the recent report, no one is proposing that Alzheimer disease can spread readily between individuals through a prion-like transmission process. However, given that aggregates of prion protein, amyloid- $\beta$ and other misfolded proteins can adhere tenaciously to stainless steel and resist degradation by standard hospital-grade sterilization processes, precaution would suggest that research and risk assessment, particularly on decontamination of medical instruments, should continue. ${ }^{12}$

\section{References}

1. Jaunmuktane Z, Mead S, Ellis M, et al. Evidence for human transmission of amyloid- $\beta$ pathology and cerebral amyloid angiopathy. Nature 2015;525:247-50.

2. Prince M, Bryce R, Albanese E, et al. The global prevalence of dementia: a systematic review and metaanalysis. Alzheimers Dement 2013;9:63-75.e2.

3. Lim J, Yue Z. Neuronal aggregates: formation, clearance, and spreading. Dev Cell 2015;32:491-501.

4. Prusiner SB. Prions. Proc Natl Acad Sci U S A 1998;95:13363-83.

5. Brown P, Brandel JP, Sato T, et al. Iatrogenic Creutzfeldt-Jakob disease, final assessment. Emerg Infect Dis 2012;18:901-7.

6. Cashman NR. Propagated protein misfolding: new opportunities for therapeutics, new public health risk. Can Commun Dis Rep 2015;41:196-9.

7. Beekes M, Thomzig A, Schulz-Schaeffer WJ, et al. Is there a risk of prion-like disease transmission by Alzheimer- or Parkinsonassociated protein particles? Acta Neuropathol 2014;128:463-76.

8. Hainfellner JA, Wanschitz J, Jellinger K, et al. Coexistence of Alzheimer-type neuropathology in Creutzfeldt-Jakob disease. Acta Neuropathol 1998;96:116-22.

9. Braak H, Braak E. Frequency of stages of Alzheimer-related lesions in different age categories. Neurobiol Aging 1997;18: 351-7.

10. Morales R, Moreno-Gonzalez I, Soto C. Cross-seeding of misfolded proteins: implications for etiology and pathogenesis of protein misfolding diseases. PLoS Pathog 2013;9:e1003537.

11. Coulthart MB, Jansen GH, Connolly T, et al. Creutzfeldt-Jakob disease mortality in Canada, 1998-2013. Can Commun Dis Rep 2015;41:183-91.

12. Thomzig A, Wagenfuhr K, Daus ML, et al. Decontamination of medical devices from pathological amyloid- $\beta$-, tau- and $\alpha$-synuclein aggregates. Acta Neuropathol Commun 2014;2:151.

Affiliations: Canadian Creuztfeldt-Jakob Disease Surveillance System (Coulthart), Public Health Agency of Canada, Ottawa, Ont.; Department of Pathology and Laboratory Medicine (Jansen), The Ottawa Hospital - Civic Campus, Ottawa, Ont.; Brain Research Centre (Cashman), University of British Columbia, Vancouver, BC

Contributors: Michael Coulthart conceived the article in discussion with Gerard Jansen and Neil Cashman and conducted the literature review. All of the authors drafted and revised the article, approved the final version to be published and agreed to act as guarantors of the work.

Acknowledgement: The authors extend their warm thanks to Alex Demarsh for sharing statistical insights. 